\title{
Endoscopic temporal ventriculocisternostomy for the management of temporal horn entrapment: report of 4 cases
}

\author{
Igor Paredes, MD, PhD, ${ }^{1}$ Javier Orduna, MD, ${ }^{2}$ David Fustero, MD, ${ }^{2}$ \\ Juan Antonio Alvarez Salgado, MD, ${ }^{3}$ Jose María Belinchon de Diego, MD, PhD, ${ }^{3}$ and \\ Francisco González-Llanos Fernández de Mesa, MD, PhD³
}

\begin{abstract}
'Department of Neurosurgery, University Hospital 12 de Octubre, Madrid; ${ }^{2}$ Department of Neurosurgery, University Hospital Miguel Servet, Zaragoza; and ${ }^{3}$ Department of Neurosurgery, University Hospital Virgen de la Salud, Toledo, Spain
\end{abstract}

\begin{abstract}
Entrapment of the temporal horn is a rare form of noncommunicating focal hydrocephalus. Standard treatment has not yet been established for this condition, and only a few cases have been reported in the literature. The authors reviewed their cases of temporal horn entrapment treated between May 2013 and December 2014 and report their experience with endoscopic temporal ventriculocisternostomy. Four patients were identified ( 3 adults and 1 child) who underwent this treatment. In 3 patients, the condition developed after tumor resection, and in 1 patient it developed after resection of an arteriovenous malformation. In 1 patient, a recurrent trapped temporal horn developed and a refenestration was successfully performed. No procedure-related complications were observed, and all of the patients remained shunt-free at last follow-up (range 4-24 months). Endoscopic temporal horn ventriculocisternostomy is a safe and effective procedure for the treatment of symptomatic temporal horn entrapment in selected cases. However, there is little experience with the procedure to recommend it as the treatment of choice.
\end{abstract}

http://thejns.org/doi/abs/10.3171/2016.1.JNS152248

KEY WORDS hydrocephalus; temporal ventriculocisternostomy; temporal horn entrapment; neuroendoscopy; trapped temporal horn

$\mathrm{T}$ EMPORAL horn entrapment is a form of noncommunicating focal hydrocephalus first described by Cairns and Daniel ${ }^{2}$ in 1947, and has been described as a consequence of penetrating trauma, meningitis, ventriculitis, intraventricular neurocysticercosis, hydatid cysts, neurosarcoidosis, intraventricular hemorrhage, intraventricular arachnoid cysts, and fibrosis after surgery for different pathologies (tumors and vascular malformations) involving the trigone of the ventricle. . $2,4,5,9,10,12,16-19$ The choroid plexus of the temporal horn continues to produce CSF, and the blockage of its outflow leads to a progressive dilation of the temporal horn. The mass effect of the dilated temporal horn over the surrounding structures produces the clinical syndrome: the compression of Meyer's loop causes homonymous hemianopia, the internal capsule compression produces hemiparesis, the hippocampal compression can cause memory disturbances, and the increased intracranial pressure (ICP) can lead to headache, gait disturbance, confusion, and even deteriorated consciousness. . $^{1,3,7,7-16,18}$ The implantation of a ventriculoperitoneal shunt ${ }^{9,18}$ was historically the treatment of choice as an alternative to open resection of the temporal tip, ${ }^{10,16}$ but less invasive procedures have been described, such as a temporal to frontal horn shunt, ${ }^{8}$ a temporal to prepontine cistern shunt, ${ }^{3,7}$ and a temporal horn ventriculocisternostomy ${ }^{11,13}$ for the management of this pathology. In this paper we report 4 cases of temporal horn entrapment treated by endoscopic temporal ventriculocisternostomy.

\section{Methods}

\section{Study Sample}

We retrospectively reviewed the cases of temporal horn entrapment treated at our hospital from May 2013 to De-

ABBREVIATIONS AChA = anterior choroidal artery; $A V M=$ arteriovenous malformation; EVD = external ventricular drain; $P C o A=$ posterior communicating artery. SUBMITTED September 27, 2015. ACCEPTED January 29, 2016.

INCLUDE WHEN CITING Published online April 15, 2016; DOI: 10.3171/2016.1.JNS152248. 
cember 2014 by temporal horn endoscopic ventriculocisternostomy. Patient demographics, medical charts, and images were retrospectively reviewed. Specific informed consent regarding the treatment as experimental was obtained from the patients or their relatives.

\section{Operative Technique}

For the operation, the patient was placed supine with the head turned to the contralateral side of the pathology. The head was either fixed with a 3-pin headholder (Mayfield) or rested on a horseshoe pillow, and a magnetic frameless neuronavigation system was used (Stealth Station, Medtronic) to design the optimal trajectory. The ideal ventriculocisternostomy site was selected on either a T1or T2-weighted, contrast-enhanced MR image, selecting the thinnest point anterior to the anterior choroidal point that avoided the optic tract and anterior choroidal artery (AChA) above, and the third cranial nerve and posterior communicating artery (PCoA) below. The entry point was selected, choosing the shortest perpendicular trajectory. Once the trajectory was planned, a small skin incision anterior and superior to the tragus was made, the temporal muscle and fascia were incised, and a $10-\mathrm{mm}$ bur hole was created. The dura mater was coagulated and opened in a cruciate fashion. Bipolar coagulation of the temporal cortex was performed and the endoscope (Richard Wolf pediatric endoscope, 6.2-mm diameter $\times 12.5-\mathrm{cm}$ length; the LOTTA endoscope was used in 1 case, $6.1-\mathrm{mm}$ diameter $\times 14-\mathrm{cm}$ length ) was introduced under navigation guidance. The whole procedure was performed under constant warm saline irrigation. Once in the temporal horn, the choroid plexus and anterior choroidal point were identified as anatomical landmarks, and a point anterior and inferior to these landmarks was selected in the temporal mesial wall with the thinnest parenchymal tissue (Fig. 1). Navigation was used to confirm optimal site selection. Low-energy bipolar coagulation was applied, followed by blunt perforation. Then a 3-Fr Fogarty balloon catheter (Edwards Lifesciences) was inserted, and the opening was enlarged by inflation of the balloon. The endoscope was progressed and the interpeduncular cistern was inspected, with extreme care taken to not damage the perforating arteries or third cranial nerve. Minor hemorrhages were addressed using copious irrigation and bipolar coagulation if necessary. Once the opening was considered sufficiently large, and a good CSF pulsation was observed through the stoma, the endoscope was removed and the bur hole was covered with a fibrin sealant patch (TachoSil, Baxter).

\section{Results}

Four patients were identified, 2 male and 2 female. Three of the patients were adults and 1 was an infant. All of the patients developed an entrapped temporal horn after a surgical procedure performed to address a vascular or tumoral pathology near or within the trigone of the lateral ventricle, and all of them as a consequence of scarring. These patients presented with symptoms between 6 weeks and 6 months after the initial surgery (mean 3.9 months). All underwent an endoscopic temporal ventriculocisternostomy. One patient required a repeat procedure,

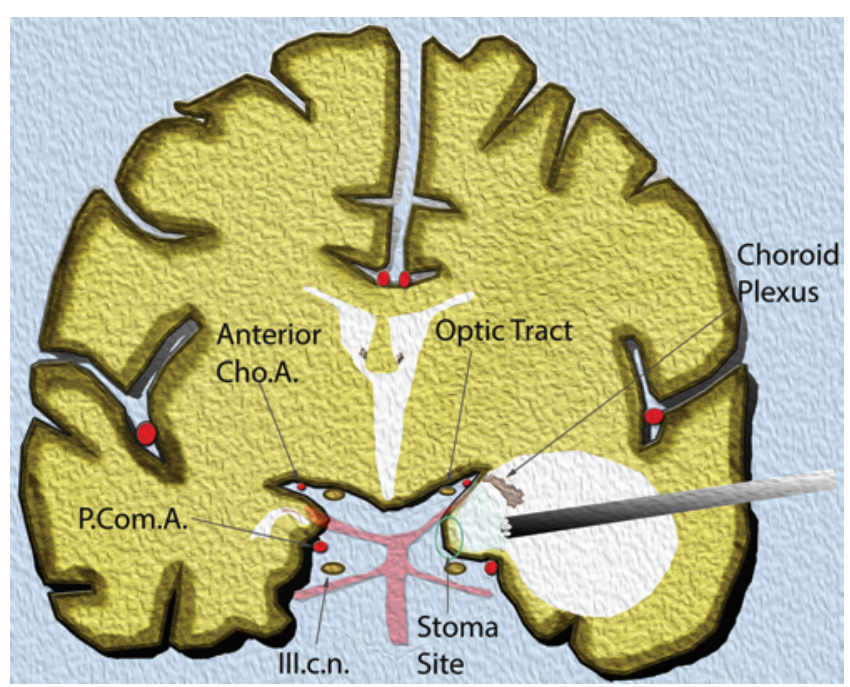

FIG. 1. Diagram showing a coronal slice at the fenestration point. The fenestration site is selected below the choroidal plexus to avoid the optic tract and AChA cranially, and the PCoA and third cranial nerve caudally. Anterior Cho.A. = anterior choroidal artery, P.Com.A. = posterior communicating artery, III.c. $n=$ third cranial nerve. Copyright Igor Paredes. Published with permission. Figure is available in color online only.

but all of the patients' entrapments were resolved, and they remained shunt-free during follow-up (mean follow-up duration 12.5 months). Three patients demonstrated immediate clinical and radiological improvement, whereas the other patient showed a rather progressive clinical and radiological improvement.

\section{Case Reports Case 1}

A 20-year-old woman presented with a sudden headache and right hemiparesis, and was diagnosed with an acute bleed ( $3 \mathrm{~cm}$ maximum diameter) of the left temporal lobe due to rupture of an arteriovenous malformation (AVM). The patient was stable and in good neurological condition, so the pathology was addressed electively a few days later. The patient was left-handed, and right hemisphere language dominance was demonstrated on functional MRI. A left temporal craniotomy was performed and the AVM was fully resected. The postoperative course was uneventful, and the patient was discharged a week later with a right-sided homonymous hemianopia and fully recovered muscle strength. Six months later the patient was admitted with a 1-month history of progressive headache and mild bilateral papilledema upon physical examination. The head MR image and CT scan showed a dilated left temporal horn. A left temporal horn ventriculocisternostomy was performed, and a closed external ventricular drain (EVD) was left in place in case an emergency CSF withdrawal was necessary. The patient's headache and papilledema resolved completely. A head CT scan was performed 48 hours later, showing marked reduction of the temporal horn, so the EVD was removed. At 6 months follow-up the patient remained symptom free, and then returned to her native country and was lost to further follow-up (Fig. 2). 

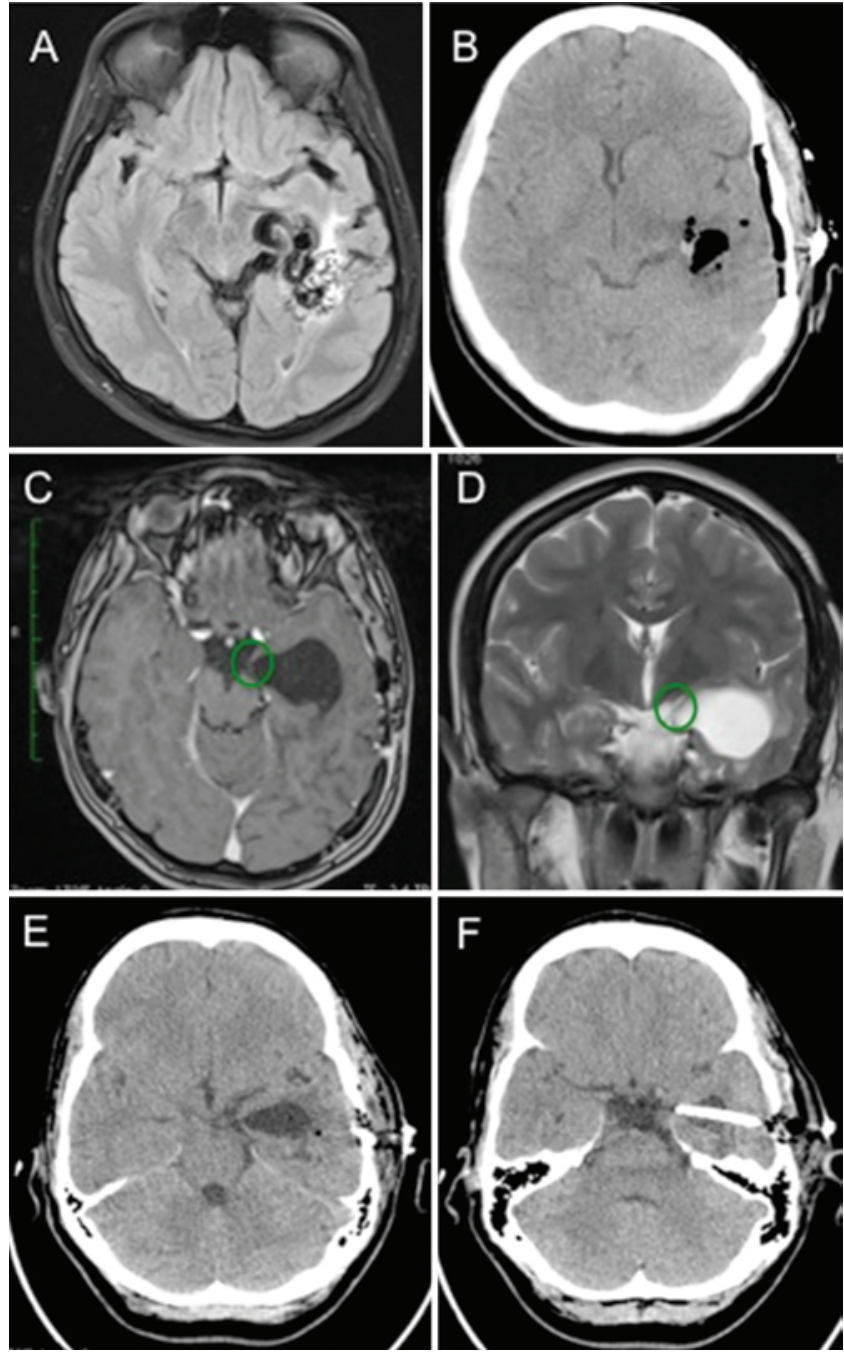

FIG. 2. Case 1. A: Axial FLAIR image showing the left temporal lobe AVM. B: Axial head CT scan after the AVM removal. C and D: Axial T1-weighted (C) and coronal T2-weighted (D) MR images showing the dilated temporal horn. The green circle indicates the selected spot chosen to perform the fenestration. E and F: Axial CT scans obtained 24 hours after the ventriculocisternostomy, showing a smaller temporal horn and a closed EVD in place. Figure is available in color online only.

\section{Case 2}

A 5-year-old girl was admitted with a 4-day history of headaches and diplopia. The CT scan showed a left intraventricular tumor situated in the atrium and temporal horn and MRI confirmed this finding. Gross-total removal of the tumor was achieved through a transparietal transcortical approach. The tumor was diagnosed as a choroid plexus carcinoma and treated accordingly with chemotherapy. Two months after surgery the patient was readmitted with a decreased level of consciousness and vomiting. The CT scan showed an entrapped left temporal horn with midline shift. An urgent temporal horn ventriculocisternostomy was performed. The neuronavigation system was not available given the emergency setting, so the procedure was performed following anatomical landmarks (Video 1).

VIDEO 1. Clip showing a left temporal horn ventriculocisternostomy performed under emergency circumstances without a navigation system available. P.C.A. = posterior cerebral artery. Copyright Igor Paredes. Published with permission. Click here to view.

A closed EVD was left in place. The postoperative CT scan showed a significant reduction of the temporal horn volume and the patient recovered well; thus, the EVD was removed. The patient was discharged 7 days after the surgery, and continued with the chemotherapy treatment of the tumor. The MR image obtained 4 months after the procedure again showed an entrapped temporal horn, suggesting closing of the stoma; therefore, the procedure was repeated. The stoma was identified, and visual confirmation of its closing was obtained. It was again reopened with the Fogarty catheter, making the stoma a bit wider. Four months after the second endoscopic surgery the patient remains asymptomatic, and MRI showed a patent reduction of the entrapped horn (Fig. 3).

\section{Case 3}

A 66-year-old man was operated on to remove a metastasis of a neuroendocrine carcinoma located at the right parietal white matter and extending to the right atrium. The patient was discharged with a left hemiparesis, but he could independently walk on his own. Six months later the patient was admitted with a left severe hemiparesis and a deteriorated mental state. CT and MRI showed a dilated right temporal horn with mass effect. A right temporal horn ventriculocisternostomy was performed. A second bur hole was made in the occipital region, and the atrium was entered with the endoscope. The fibrosis was bluntly punctured, but good communication with the temporal horn was not achieved. The patient improved after surgery, partially recovering his strength on the left side and regaining a normal mental status. The CT scan showed a reduction in the temporal horn. The patient was discharged home and he continued to improve with physical therapy. He remained shunt-free until he died 2 years later of progression of his underlying disease (Fig. 4).

\section{Case 4}

A 73-year-old man underwent an operation for an epidermoid carcinoma of the vocal cord. During the extension of the disease screening, a mass was found in the right temporal atrium. Although no other lesion was found, a metastasis of the initial carcinoma was believed to be the most probable diagnosis, so it was removed through a transparietal route. The mass was finally diagnosed as a tuberculoma secondary to an atypical mycobacterium $(M$. scrofulaceum). The patient was discharged with a mild superior left homonymous quadrantanopia. Six weeks later the patient was readmitted with a left hemiparesis and left homonymous hemianopia. CT and MRI showed a right dilated temporal horn. A temporal ventriculocisternostomy was performed (Video 2).

VIDEO 2. Clip showing a right temporal horn ventriculocisternostomy performed under scheduled circumstances with a navigation system available. P.C.A. = posterior cerebral artery. Copyright Igor Paredes. Published with permission. Click here to view.

The neurological deficits slowly improved in the patient. He was treated with antitubercular agents for 9 months 

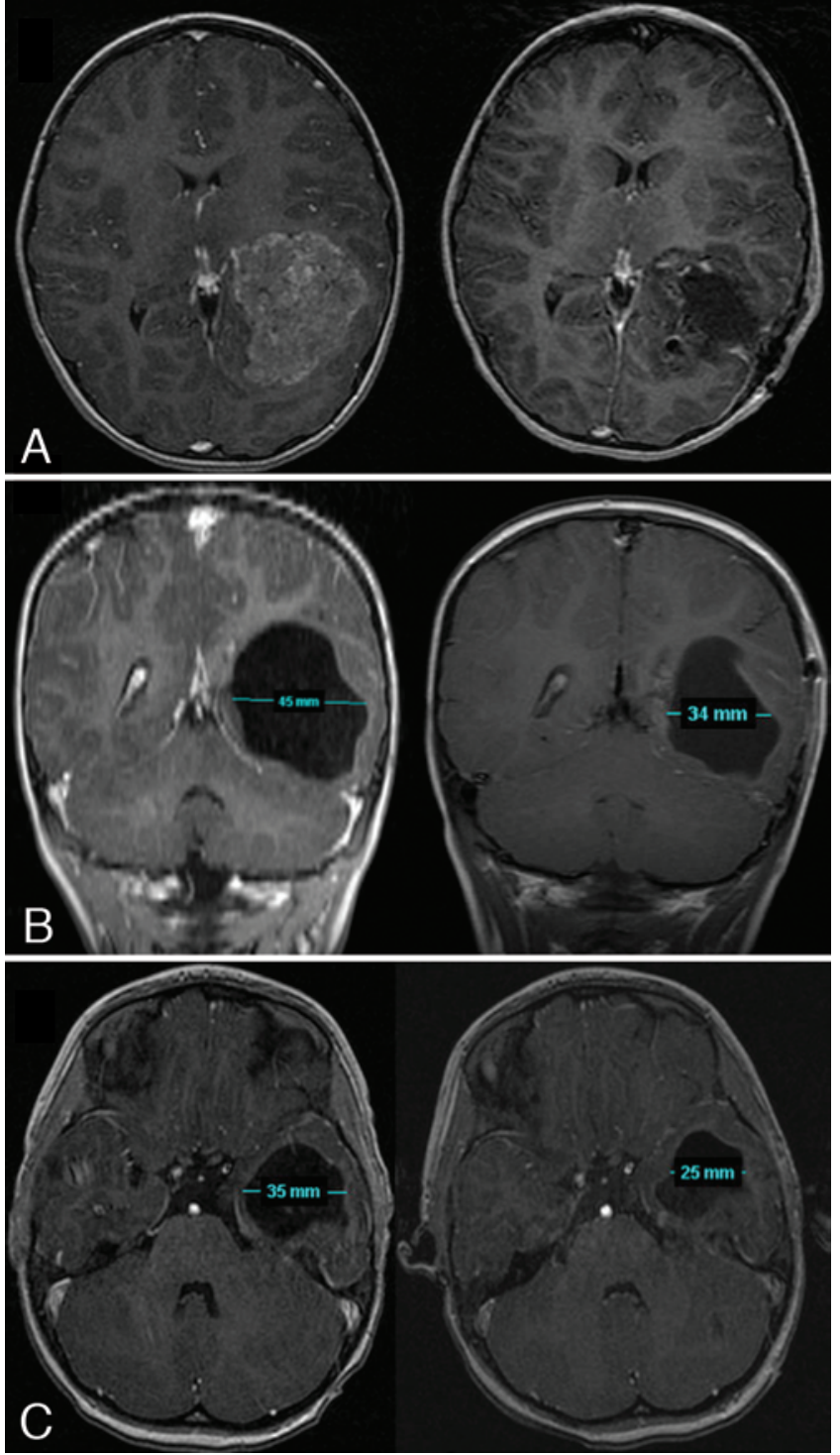

FIG. 3. Case 2. A: Axial T1-weighted postcontrast-enhanced MR images showing the tumor in the left atrium (left), and a control image after surgery (right). B: Coronal T1-weighted postcontrast-enhanced MR images showing the dilated temporal horn (left), and control image after the fenestration (right). Diameters are shown to compare sizes. C: Axial T1-weighted postcontrast-enhanced MR images showing the dilated left temporal horn (left), and control image after the ventriculocisternostomy showing shrinkage of the temporal horn (right). Diameters are depicted to compare sizes. Figure is available in color online only.

after the surgery. In this case the MR image did not show an immediate reduction in the temporal horn volume, but a rather progressive shrinkage that was obvious at 3 and 6 months of follow-up. The patient remained shunt-free at the 1-year follow-up evaluation (Fig. 5). Given the concurrent atypical tuberculosis, it was uncertain if the improvement was due to the surgical procedure, the antitubercular agents, or the combination of both therapies.

\section{Discussion}

Temporal horn entrapment might be a radiological
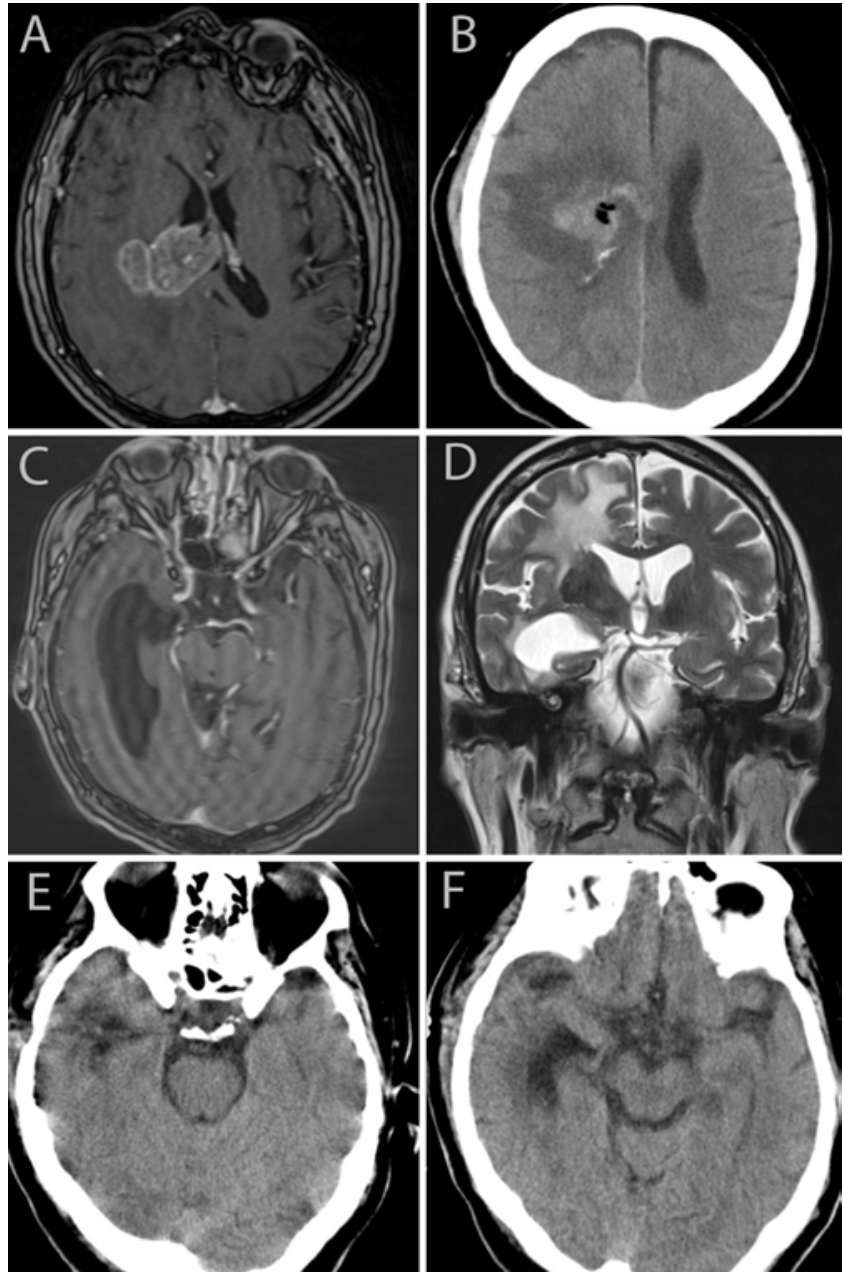

FIG. 4. Case 3. A: Axial postcontrast-enhanced T1-weighted MR image showing the right atrium with the tumor. B: Axial CT scan after tumor removal. C: Axial T1-weighted MR image showing the dilated right temporal horn. D: Coronal T2-weighted MR image showing the dilated temporal horn. E and F: Axial head CT scans obtained 24 hours after the ventriculocisternostomy, showing shrinkage of the temporal horn.

finding, or it might present as a combination of contralateral homonymous hemianopia, contralateral hemiparesis, memory loss, and symptoms of raised ICP. ${ }^{1-18}$ The presence of these symptoms precludes an observational attitude, and compels one to seek a rapid solution. Different options are available to divert the CSF away from the temporal horn, like shunting it to the frontal horn or to the peritoneal cavity. $3,8,12,18$ Nevertheless, we strongly believe that shunt placement or implantation of foreign bodies should be avoided because of the risk of infection or failure, necessitating future revisions. ${ }^{6}$ Whenever possible, reconnection to the CSF pathway should be attempted, either following its normal path to the atrium, ${ }^{5}$ or bypassing it to the subarachnoid space through a surgical opening of the choroidal fissure..$^{11,13}$

The endoscopic dilation of the scarred trigone that blocks the CSF pathway is an option, but the surgical opening of a scar carries the obvious risk or re-scarring, leading to a relapse in the temporal horn entrapment. ${ }^{7}$ 

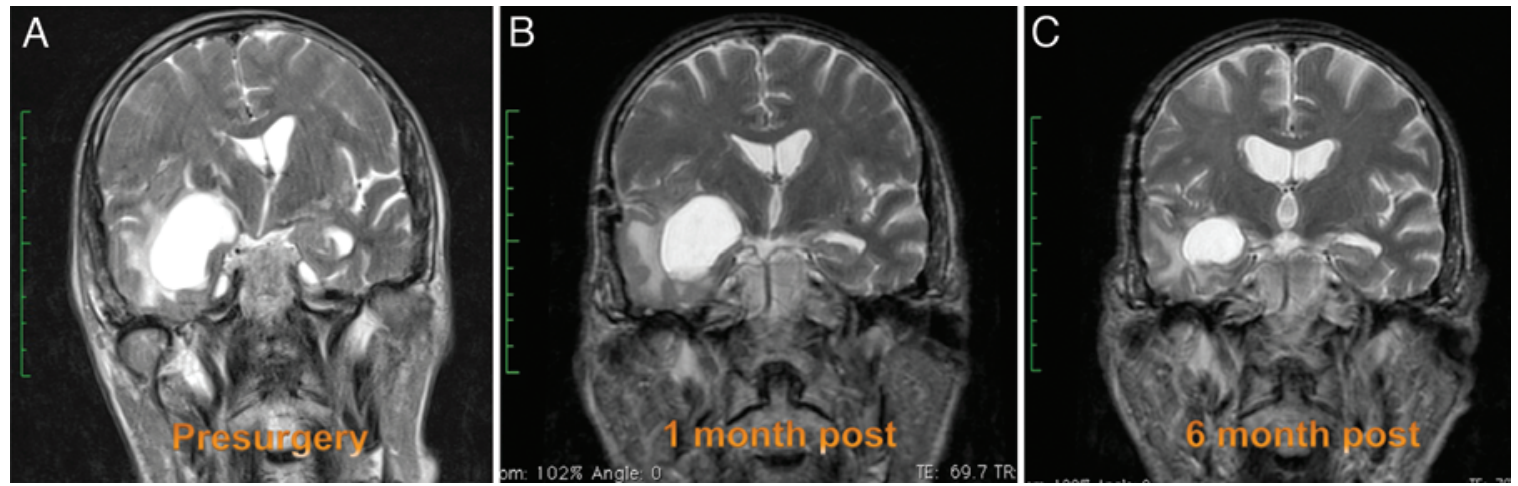

FIG. 5. Case 4. A: Coronal T2-weighted MR image showing the dilated right temporal horn. B and C: Coronal T2-weighted MR images demonstrating progressive shrinkage of the temporal horn 1 and 6 months after the ventriculocisternostomy, respectively. Figure is available in color online only.

The endoscopic ventriculocisternostomy is a safe procedure, and can be performed in the setting of a malignancy or an infection (as in 1 of our cases) because there is no risk of seeding the malignant cells away from the CNS, or suffering from a shunt infection that would need a revision surgery. The anatomy of the region is usually quite distorted by the dilation of the temporal horn, thus making the procedure difficult based only on anatomical landmarks. In addition, there are important structures in the mesial wall of the temporal horn that need to be avoided, leaving a relatively narrow window to safely perform the ventriculocisternostomy. The dilation of the temporal horn widens the window between the AChA and optic track superiorly, and the PCoA and third cranial nerve inferiorly, making the procedure feasible. Only when that window is wide enough and the mesial wall thin enough should the procedure be attempted. Otherwise, the procedure should not be considered safe, and alternative treatment should be offered to the patient. Even when the window is wide and the mesial wall thin, we believe the use of neuronavigation is essential to design the safest trajectory. The structures mentioned should be identified in a coronal T2-weighted MR image to find the safest axial plane in between them, and then choose the ventriculocisternostomy point within that plane on axial T1- or T2-weighted thin-slice MR images. Next, the shortest trajectory from the cortex is chosen, usually leading to an entry point in the middle temporal gyrus (Fig. 1). Our own initial lack of confidence in the procedure led us to leave a closed EVD in place in our first and second cases, and to try endoscopic reopening of the trigone scarring in the third case. The EVDs were not needed, and the endoscopic reopening of the trigone was disappointing, because the thickness of the scar made it unfeasible. For our fourth case we performed only the ventriculocisternostomy, which was equally effective.

In this paper we presented a series of 4 patients treated with temporal horn ventriculocisternostomy in which it was effective and no procedure-related complications were encountered. This is a small case series, and we lost 1 of the patients to follow-up at 6 months, so no strong recommendations can be made. There are other surgical options to be considered, such as different shunting procedures, and there are no large studies comparing them that would allow us to favor one procedure over another. Also, asymptomatic temporal horn entrapments might be closely observed without any treatment. The decision on what treatment to apply should be based on individual anatomy, underlying pathology, available techniques, and the experience of the treating surgeon.

Endoscopic temporal horn ventriculocisternostomy is a safe and effective procedure for the treatment of symptomatic temporal horn entrapment in selected cases. However, there is little experience with the procedure to recommend it as the treatment of choice. Further investigation is needed to choose the best treatment for this condition.

\section{References}

1. Berhouma M, Abderrazek K, Krichen W, Jemel H: Apropos of an unusual and menacing presentation of neurosarcoidosis: the space-occupying trapped temporal horn. Clin Neurol Neurosurg 111:196-199, 2009

2. Cairns H, Daniel P: Localized hydrocephalus following penetrating wounds of the ventricle. Br J Surg 55 (Suppl 1):187-197, 1947

3. Chen CC, Kasper EM, Zinn PO, Warnke PC: Management of entrapped temporal horn by temporal horn to prepontine cistern shunting. World Neurosurg 79:404.e7-404.e10, 2013

4. Coria F, Bahíllo Marcos E, Moral Blanco M, García Gutiérrez P, Ortiz Sáenz de Santa María R: Late-onset isolated gelastic epilepsy secondary to entrapment of the right temporal horn. Neurologia 15:204-207, 2000

5. Ellis JA, McCormick PC II, Feldstein NA, Ghatan S: Transoccipital endoscopic fenestration of atrial cysts causing ventricular entrapment. J Neurosurg Pediatr 15:567-572, 2015

6. Gliemroth J, Käsbeck E, Kehler U: Ventriculocisternostomy versus ventriculoperitoneal shunt in the treatment of hydrocephalus: a retrospective, long-term observational study. Clin Neurol Neurosurg 122:92-96, 2014

7. Hana T, Tanaka S, Shin M, Mukasa A, Kugasawa K, Saito N: Neuroendoscopic ventriculocisternostomy with stent placement for trapped temporal horn after the resection of glioblastoma. World Neurosurg 84:2078.e5-2078.e8, 2015

8. Hervey-Jumper SL, Ziewacz JE, Heth JA, Sullivan SE: Frontal-to-temporal horn shunt as treatment for temporal horn entrapment. J Neurosurg 112:410-413, 2010

9. Iaccarino C, Romano A, Ramponi V, Nasi D, Maggi M, Pascarella R, et al: Entrapment of temporal horn: first case of bilateral occurrence and review of literature. Clin Neurol Neurosurg 115:2207-2212, 2013 
10. Kamali NI, Huda MF, Srivastava VK: Intraventricular hydatid cyst causing entrapped temporal horn syndrome: Case report and review of literature. Trop Parasitol 1:113-115, 2011

11. Krähenbühl AK, Baldauf J, Gaab MR, Schroeder HW: Endoscopic temporal ventriculocisternostomy: an option for the treatment of trapped temporal horns. J Neurosurg Pediatr 11:568-574, 2013

12. Maurice-Williams RS, Choksey M: Entrapment of the temporal horn: a form of focal obstructive hydrocephalus. J Neurol Neurosurg Psychiatry 49:238-242, 1986

13. Parrent AG: Endoscopically guided fenestration of the choroidal fissure for treatment of trapped temporal horn. J Neurosurg 93:891-894, 2000

14. Schroeder HW, Oertel J, Gaab MR: Endoscopic treatment of cerebrospinal fluid pathway obstructions. Neurosurgery 62 (6 Suppl 3):1084-1092, 2008

15. Sharma C, Acharya M, Kumawat BL, Kochar A: 'Trapped temporal horn' of lateral ventricle in tuberculous meningitis. BMJ Case Rep 2014:bcr2014203837, 2014

16. Singh SK, Srivastava C, Ojha BK, Chandra A, Parihar A, Husain N: An unusual cause of entrapment of temporal horn: Neurocysticercosis. Neurol India 58:814-815, 2010

17. Smith H, Moody D, Ball M, Laster W, Kelly DL Jr, Alexander E Jr: The trapped temporal horn: a trap in neuroradiological diagnosis. Neurosurgery 5:245-249, 1979

18. Tsugane R, Shimoda M, Yamaguchi T, Yamamoto I, Sato O: Entrapment of the temporal horn: a form of focal non-communicating hydrocephalus caused by intraventricular block of cerebrospinal fluid flow--report of two cases. Neurol Med Chir (Tokyo) 32:210-214, 1992
19. Watanabe T, Katayama Y: Evaluation by magnetic resonance imaging of the entrapped temporal horn syndrome. J Neurol Neurosurg Psychiatry 66:113, 1999

\section{Disclosures}

The authors report no conflict of interest concerning the materials or methods used in this study or the findings specified in this paper.

\section{Author Contributions}

Conception and design: Paredes, Alvarez Salgado. Acquisition of data: Paredes, Orduna, Fustero, Alvarez Salgado, Belinchon de Diego. Analysis and interpretation of data: Paredes, Orduna, Alvarez Salgado, Belinchon de Diego. Drafting the article: Paredes. Critically revising the article: Orduna, González-Llanos Fernández de Mesa. Approved the final version of the manuscript on behalf of all authors: Paredes. Administrative/technical/ material support: Paredes. Study supervision: González-Llanos Fernández de Mesa.

\section{Supplemental Information \\ Videos}

Video 1. https://vimeo.com/155835887.

Video 2. https://vimeo.com/155835946.

\section{Correspondence}

Igor Paredes, Hospital 12 de Octubre, Avenida de Córdoba s/n, Madrid 28041,Spain. email: igorparedes@gmail.com. 